\title{
Environmental groups in monopolistic markets
}

\author{
Pim Heijnen · Lambert Schoonbeek
}

Received: 9 June 2005 / Accepted: 3 April 2007 / Published online: 5 June 2007

(C) Springer Science+Business Media B.V. 2007

\begin{abstract}
We examine a market in which a monopolistic firm supplies a good. The production of the good causes damage to the environment. Consumers are heterogeneous with respect to their disutility of the environmental damage. An environmental group can enter the market and set up a campaign in order to influence consumers' preferences. We characterize the equilibrium of the resulting entry-deterrence game and investigate its properties. It turns out that the aggregated environmental damage is lowest if the firm is able to deter entry of the environmental group and, moreover, the fixed entry cost of the environmental group is small enough.
\end{abstract}

Keywords Environmental group $\cdot$ Environmental quality $\cdot$ Monopolist $\cdot$ Entry deterrence JEL classification $\mathrm{L} 1 \cdot \mathrm{Q} 2$

\section{Introduction}

Environmental groups (EGs) use campaigns in order to communicate with consumers. ${ }^{1}$ One of the goals of such campaigns can be to discourage people from buying certain goods, because the production process of these goods generates too much damage or pollution to the environment. For example, it can happen that consumers are aware of the environmental damage caused by the consumption of a certain good and dislike this detrimental effect. Yet,

1 For example, Greenpeace organizes campaigns about the environmental damage caused by different consumer products, see under 'campaigns' at www.greenpeace.org.uk.

P. Heijnen $(\bowtie)$

CenDEF and Department of Quantitative Economics, University of Amsterdam, Roetersstraat 11, 1018 WB Amsterdam, The Netherlands

e-mail: P.Heijnen@uva.nl

L. Schoonbeek

Department of Economics, University of Groningen, P.O. Box 800, 9700 AV Groningen, The Netherlands e-mail: L.Schoonbeek@rug.nl 
a campaign launched by an EG could increase the level of guilt felt by them if they purchase the good in question.

This paper investigates a model that can be used to study the effects of such a campaign of an EG. In our model we consider a profit-maximizing monopolistic firm that supplies a good on a market. The production of this good causes damage to the environment. The monopolist can control the environmental damage generated per-unit of production by choosing an appropriate production technique. However, the use of a cleaner technique also implies that the per-unit cost of production becomes higher. Utility-maximizing consumers dislike environmental damage, but they are heterogeneous in this respect (for empirical evidence that consumers care about the environment, see e.g., Bjørner et al. 2004; Brockman et al. 1996; Teisl et al. 2002). The key feature of the model is that an EG can decide to enter the market and expend effort in a campaign aimed at increasing each consumer's disutility of the environmental damage that results if he or she consumes the good. The ultimate goal of the EG is to minimize the aggregated environmental damage (expressed in monetary value) plus the costs of their campaign.

Our model is a three-stage game in which first the monopolist selects the level of environmental damage per-unit of production, next the EG decides whether it will enter and expend effort in a publicity campaign, and finally the monopolist chooses the price of its product and the consumers decide whether to buy the good. We derive the (subgame-perfect Nash) equilibrium of the model and discuss its properties. More particularly, we demonstrate that the monopolist and EG are involved in a so-called entry-deterrence game (for a general discussion of such games, see Tirole 1988, pp. 314-317). That is, in equilibrium we can have a situation with either blockaded entry, or entry deterrence or entry accommodation between the monopolist and EG. We characterize, in terms of the size of the fixed entry cost of the $\mathrm{EG}$, which of these three situations is relevant in equilibrium. Moreover, we compare the size of the aggregated environmental damage across these situations. We show that aggregated damage is highest if entry is blockaded. When entry is deterred, aggregated damage obviously is lower than under blockaded entry. Under entry deterrence, the monopolist deliberately selects a production technique that is clean enough such that it is not profitable for the EG to enter and start a campaign. ${ }^{2}$ Interestingly, for a range of small enough values of the EG's fixed entry cost, entry deterrence also yields lower aggregated damage than entry accommodation. The mere threat of a campaign by the EG then disciplines the firm most effectively to supply a relatively clean good. We remark that our analysis also provides a rationale for why a government might want to subsidize EGs: by doing so, it can try to get the entry cost of an EG in the relevant range. ${ }^{3}$

Our model is closely related to the vertical product differentiation duopoly model with environmentally conscious consumers presented by Arora and Gangopadhyay (1995) and Moraga-González and Padrón-Fumero (2002). Further references that elaborate on the latter type of model are Bansal and Gangopadhyay (2003) and Cremer and Thisse (1999). We remark here that these studies focus on the effectiveness of traditional tax and regulatory measures a government might use in order to reduce environmental damage. Non-governmental organizations like an EG do not play any role in these studies. Instead, our study focuses

\footnotetext{
2 It is interesting to observe here that the electronics company Samsung announced in 2004 that it will replace toxic chemicals (like brominated flame retardants) in some of its products by safer alternatives. This decision was made after initial talks with Greenpeace (see www.greenpeace.org.uk). We can interpret Samsung's behavior as an attempt to deter a campaign by this EG.

${ }^{3}$ We notice that in practice governments subsidize EGs. For example, the Dutch Ministry of Housing, Spatial Planning and the Environment provides such subsidies through the program SMOM, see www.vrom.nl/pagina.html?id=9813.
} 
precisely on the strategic effects between this type of organization and an industry. Doing so, we limit attention to the most simple form of an industry, i.e., an industry with a monopolistic firm. This is done for reasons of analytical tractability, and also enables us to ignore strategic effects that might exist between firms in an industry.

We can make three further remarks concerning related literature. First, Liston-Heyes (2001) also examines a model in which a monopolistic firm and an EG have conflicting interests. However, different from our approach, in her model the firm needs permission from the government for a project which, if approved, yields profit to the firm but damage to the environment. The EG is opposed to the project. The decision by the government is binary: either the firm is allowed to carry out its plan, or the permission is denied. The situation is modelled as a rent-seeking contest in which both players lobby at the government in order to increase the probability of a decision which is favourable from their own perspective. Related to the approach in our model, Liston-Heyes assumes that the EG wants to minimize the expected environmental damage costs plus lobbying expenditures. For a similar model, see also Heyes (1997).

Second, Maxwell et al. (2000) discuss a three-stage model in which identical Cournot oligopolists face the possibility of mandatory pollution abatement regulation by the government. The firms first select a voluntary abatement level. Next, the consumers observe this abatement level and determine whether to enter a so-called influence game. Finally, the firms play a Cournot production game. If the consumers enter the influence game, then they as well as the firms exert pressure on the government in order to induce it to prescribe a level of abatement that is desirable from their own perspective, respectively, and in turn the mandatory abatement policy is determined by the government. It turns out that, just like in our model, in equilibrium there are circumstances such that the firms deter entry of the influence game by selecting an appropriate (high-enough) voluntary abatement level. However, contrary to Maxwell et al., governmental regulation does not play a role in our model.

Third, we observe that our analysis is related to the theoretical industrial organization literature on advertising models. In this literature one usually makes a distinction between so-called persuasive advertising, where a firm attempts to shift consumers' preferences in favour of its product (e.g., think of an advertisement that emphasizes that 'you are cool when you drink this beverage'), and informative advertising, where a firm provides consumers information about basic attributes of its product (e.g., about its price). Both types of advertising are relevant in practice, and they are extensively studied in the theoretical (and empirical) advertising literature; see Bagwell (2005), Carlton and Perloff (2005) and Tirole (1988). Clearly, we can make a similar distinction with regard to campaigns of an EG: they can be either persuasive or informative. In this paper we focus on the former type. We have a market with consumers which are heterogeneous with respect to their environmental consciousness, and the campaign of the EG is considered as 'negative persuasive advertising', i.e., it is designed to shift each consumer's taste by increasing the uneasiness felt if the consumer consumes the damaging good (e.g., think of a campaign against the use of fur that shows pictures of baby seals which are bloodily clubbed to death). ${ }^{4}$ For related studies in the standard advertising literature on persuasive advertising in markets with heterogeneous consumers, see e.g., Bloch and Manceau (1999), von der Fehr and Stevik (1998) and Tremblay and Martins-Filho (2001).

\footnotetext{
${ }^{4}$ Levitt and Dubner (2005, p. 152) discuss environmental risks in terms of an objective hazard and subjective outrage felt by the public. They interpret activities of EG's as 'outrage increase', hence negative persuasive advertising in our terminology.
} 
Of course, besides persuasive campaigns of an EG, it is also interesting to investigate campaigns which are informative. This might be relevant if for example we have situations with asymmetric information, where the EG and firm have full information about the true damage generated by the consumption of a good, whereas consumers do not have this information. In a related paper, Heijnen (2007) investigates the effects of an informative campaign of an EG in such a situation, using a model that is basically the same as used here. That study takes into account that the EG has an incentive to exaggerate the amount of damage caused by the good, whereas the firm has the opposite incentive (for a comprehensive account of problems related to the transmission of information by special interest groups like EGs, see Grossman and Helpman 2001). We remark here that the analysis in Heijnen (2007) is entirely different than here, and boils down to an investigation of signalling equilibria, where the EG and firm try to signal the environmental quality of the good by selecting an appropriate level of, respectively, the costs of the campaign and the price of the good. For related studies in the standard advertising literature, see e.g., Hertzendorf and Overgaard (2001) and Milgrom and Roberts (1986).

The paper proceeds as follows. In Sect. 2 we introduce the model. In Sect. 3 we derive the equilibrium, and in Sect. 4 we examine some interesting implications of the equilibrium. Section 5 concludes. Proofs and technical details are in two Appendices.

\section{The model}

Consider a market with a profit-maximizing monopolistic firm which supplies one good. The production of each unit of the good generates a damage, $d>0$ say, to the environment. The actual size of $d$ depends on the production technique chosen by the firm. Once this technique has been chosen, the marginal (production) cost for the firm is constant and given by the function $c(d)>0$. We assume that $c^{\prime}(d)<0$ and $c^{\prime \prime}(d)>0$ for all $d$. If a good is produced such that more environmental damage is caused, it also becomes cheaper to produce this good, but this effect diminishes for larger $d$. The firm has no fixed cost.

Consumers buy either one unit of the good or do not buy it. They are uniformly distributed on the line $[0, \bar{\theta}]$ with mass one, where $\bar{\theta}>0$ is given. A consumer $\theta \in[0, \bar{\theta}]$ receives a utility $U=V-\theta d-p$ if he buys the good and zero otherwise. The good is only bought if $U \geq 0$. The term $V>0$ denotes the intrinsic utility of the good, $\theta d$ is the disutility experienced by consumer $\theta$ as a consequence of the environmental damage generated by the production of the good, and $p$ is the price of the good. Note that consumers are heterogeneous in the disutility they experience from environmental damage.

An EG has the option to enter this market, i.e., it can set up a campaign in which it exerts effort to change consumers' preferences by making consumers feel more uneasy with the environmental damage caused by the good. Denoting the EG's effort as $x$, we have $x>0$ if the EG enters and $x=0$ otherwise. We assume that as a result of a positive effort $x$, the preference parameters shift from $\theta$ to $\theta+x$ for all $\theta$, i.e., the disutility of environmental damage rises with $x d$ (see e.g., von der Fehr and Stevik 1998, p. 117, for a similar approach in a standard advertising model). For the EG, it is costly to exert effort; the corresponding cost function is given by $s(x)$, with $s(x)>0, s^{\prime}(x)>0$ and $s^{\prime \prime}(x)>0$ for all $x>0$, and $s(0)=s^{\prime}(0)=s^{\prime \prime}(0)=0$. We call $s(x)$ the publicity cost of the EG. The EG also has to pay a sunk entry cost $F \geq 0$, but only if $x>0$. The entry cost can be viewed as the nonrecoverable fixed cost of, for example, establishing contact with the media or recruiting volunteers, i.e., all things necessary to set up a campaign. The EG tries to minimize its total costs. If it enters, these costs are given by $\gamma q d+s(x)+F$, where $q$ denotes the demand for 
the firm's good, $q d$ is the aggregated environmental damage caused by the market demand, and the parameter $\gamma>0$ converts environmental damage to its monetary value. If the EG does not enter, its total costs are equal to $\gamma q d$.

We now consider a game with the following timing: in $t=1$ the firm chooses $d$ (by selecting a corresponding production technique); in $t=2$ the EG chooses whether to enter or not. After entry the EG chooses $x>0$, otherwise $x=0$; in $t=3$ the firm selects $p$ and next the consumers decide whether to buy the good or not. The timing of the game reflects two concerns. For the firm it is relatively difficult to change its production process, but it is easy to change the price. While the firm may change the price as a response to the campaign of the EG, in the short run it is unlikely that it can change the production process.

In the next section we will derive the equilibrium of the game by using backward induction. We assume that there always must be some consumers who are willing to purchase the firm's good, even if the EG has entered and exerted effort. Basically, this means that the EG is not so influential that it can completely sweep away the firm's demand. On the other hand, we also assume that there are always some consumers who are not willing to buy the good. In other words, the market is never covered, even if the EG does not enter. If the market would be covered in that case, then it is likely that the market would remain covered for small values of $x$, which would imply that then total demand remains constant. In this sense, a covered market is a barrier to entry since the EG has to exert considerable effort to have a minimal impact on demand. This would complicate our analysis without adding additional insight. The assumption of an uncovered market is standard in the literature, see e.g., also Moraga-González and Padrón-Fumero (2002).

Finally, if below a concrete functional form for $c(d)$ or $s(x)$ is needed, then we choose, respectively, $c(d)=\alpha / d^{2}$ and $s(x)=\beta x^{2}$, with $\alpha>0$ and $\beta>0$. We notice that this specification for $c(d)$ is also used by Moraga-González and Padrón-Fumero (2002). It turns out that we do not need these functional forms in the analysis of stages 2 and 3; however, we do need them in our analysis of stage 1 .

\section{The equilibrium of the model}

\subsection{Stage 3}

To begin with, suppose that the EG has entered and that $x>0$ as well as $d>0$ are given. We want to solve for the corresponding optimal price in stage 3. Observe first that in this case the consumers are located on the line $[x, \bar{\theta}+x]$. The indifferent consumer is found by solving $U=0$ for $\theta$, which gives $\theta=(V-p) / d$. As a result, demand is given by

$$
q=\frac{V-p}{\bar{\theta} d}-\frac{x}{\bar{\theta}}
$$

and profit equals

$$
\Pi=[p-c(d)]\left[\frac{V-p}{\bar{\theta} d}-\frac{x}{\bar{\theta}}\right] .
$$

The first-order condition of profit-maximization reads

$$
\frac{\mathrm{d} \Pi}{\mathrm{d} p}=\frac{V-p}{\bar{\theta} d}-\frac{x}{\bar{\theta}}-\frac{p-c(d)}{\bar{\theta} d}=0,
$$


which gives the optimal price

$$
p^{e}=\frac{V+c(d)-x d}{2},
$$

where the superscript ' $\mathrm{e}$ ' denotes that we consider the case of entry. Notice that $\mathrm{d}^{2} \Pi / \mathrm{d} p^{2}=$ $-2 /(\bar{\theta} d)<0$, which shows that $p^{e}$ indeed gives a maximum.

We easily obtain the following expressions for, respectively, the demand and profit in the case of entry:

$$
\begin{gathered}
q^{e}=\frac{V-c(d)-x d}{2 \bar{\theta} d}, \\
\Pi^{e}=\frac{[V-c(d)-x d]^{2}}{4 \bar{\theta} d} .
\end{gathered}
$$

We assume throughout the paper that, given $x$ and $d$, demand is positive and the market not covered. A necessary and sufficient condition for this to hold is:

Assumption $10<V-c(d)-x d<2 \bar{\theta} d$.

Notice that Assumption 1 also guarantees that the firm's profit $\Pi^{e}$ is positive. Concluding our discussion of the case of entry, we notice that, in fact, $p^{e}, q^{e}$ and $\Pi^{e}$ are functions of $x$ and $d$. In order to simplify the notation, we do not mention this dependency explicitly.

Next, let us turn to the case where the EG has not entered (and thus $x=0$ ), and suppose that $d>0$ is given. In order to find for this case with no entry the optimal price, and the corresponding indifferent consumer, demand and profit, we can simply substitute $x=0$ in the results derived above for the case with entry. Using obvious notation, we then obtain from, respectively, (4), (5) and (6) the following expressions:

$$
\begin{array}{r}
p^{n e}=\frac{V+c(d)}{2}, \\
q^{n e}=\frac{V-c(d)}{2 \bar{\theta} d}, \\
\Pi^{n e}=\frac{[V-c(d)]^{2}}{4 \bar{\theta} d} .
\end{array}
$$

Observe that Assumption 1 is sufficient to guarantee that $0<q^{n e}<1$. Also notice that $p^{n e}$, $q^{n e}$ and $\Pi^{n e}$ are functions of $d$. Again, we do not mention this dependency explicitly.

\subsection{Stage 2}

\subsubsection{The decision of the $E G$}

Turning to stage 2, suppose that $d>0$ is given. The EG now has to choose between two options: either it enters and chooses an effort level $x>0$, or it does not enter (and then $x=0$ ). In making its decision, the EG takes into account the subsequent behaviour of the firm and consumers in stage 3 as discussed above. First, suppose that the EG enters. The EG then has to determine its optimal effort $x$ by minimizing its total costs $\gamma q^{e} d+s(x)+F$ with respect to $x$. This leads to the first-order condition

$$
s^{\prime}(x)=\frac{\gamma d}{2 \bar{\theta}},
$$


which has a unique solution, $x^{e}>0$ say, given the assumptions we made with respect to the function $s(x)$. The second-order condition for a minimum is satisfied, since $s^{\prime \prime}(x)>0$ for all $x>0$. Clearly, $x^{e}$ is a function of $d$, i.e., in fact $x^{e}=x^{e}(d)$. However, in order to save on the notation, we simply write $x^{e}$ unless it is more convenient to do otherwise. We implicitly also assume that $V-c(d)-x^{e} d>0$, i.e., Assumption 1 must be satisfied. Remark that in the case with $s(x)=\beta x^{2}$, we have $x^{e}=\gamma d /(4 \beta \bar{\theta})$.

It is obvious that the EG will decide to enter if and only if

$$
\gamma q^{e} d+s\left(x^{e}\right)+F<\gamma q^{n e} d
$$

where $q^{e}$ follows from (5) by substituting $x=x^{e}$. We can present the following result.

Proposition 1 Assume that $d>0$ is given. Then there exists an $\hat{F}(d)>0$ such that for $0 \leq F<\hat{F}(d)$ the EG enters and exerts effort $x^{e}(d)$ which solves $(10)$, whereas for $F \geq \hat{F}(d)$ the EG does not enter. Moreover, $\hat{F}(d)$ is increasing in $d$.

Proof See Appendix A.

Hence, the EG only enters if $F$ is smaller than a unique and positive threshold $\hat{F}(d)$.

For the case with $s(x)=\beta x^{2}$, we can find an explicit expression for $\hat{F}(d)$, by noting that in this case

$$
\begin{aligned}
\hat{F}(d) & =\frac{\gamma x^{e} d}{2 \bar{\theta}}-s\left(x^{e}\right) \\
& =\frac{\gamma}{2 \bar{\theta}}\left(\frac{\gamma d}{4 \beta \bar{\theta}}\right) d-\beta\left(\frac{\gamma d}{4 \beta \bar{\theta}}\right)^{2} \\
& =\frac{\gamma^{2} d^{2}}{16 \beta \bar{\theta}^{2}}>0
\end{aligned}
$$

\subsection{Stage 1}

Let us now consider the firm's choice of $d$ in stage 1, given the subsequent behaviour analysed above for stages 2 and 3 . In order to obtain tractable results, we use throughout the remainder of the paper the functional forms $c(d)=\alpha / d^{2}$ and $s(x)=\beta x^{2}$.

In stage 1 , the firm has to choose $d$ while facing possible entry of the EG. In fact, it turns out that the following three things can happen: blockaded entry, entry deterrence and entry accommodation. In the first two cases entry is barred, but for different reasons. If the firm ignored the threat of entry, then it would set environmental damage such that its profit were maximized given $x=0$. In the case of blockaded entry it can simply ignore the EG without further consequences. In the case of entry deterrence, however, the threat of entry is credible and if the firm does not lower its level of environmental damage, the EG would enter. The firm does lower environmental damage (because in this case it is profitable to do so) and discourages the EG to enter. In the case of entry accommodation, the firm could possibly still deter entry, but it is more profitable to let the EG enter and anticipate this entry.

It turns out that our model has a unique equilibrium. More particular, let us denote $d^{*}(F)$ as the equilibrium value of the environmental damage per-unit of production as a function of $F$. Further, let $d^{a c c}$ denote the optimal value of the environmental damage if the firm accommodates, and $d^{\text {block }}$ the optimal value of environmental damage in case of blockaded entry. Using this notation, we obtain the following proposition. 


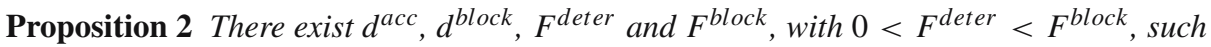
that the following holds in the equilibrium:

(i) If $0 \leq F<F^{\text {deter }}$, then the firm chooses $d^{*}(F)=d^{\text {acc }}$, and the EG enters and behaves as described in Proposition 1.

(ii) If $F^{\text {deter }} \leq F<F^{\text {block }}$, then the firm chooses $d^{*}(F)=\hat{d}(F) \equiv \sqrt{16 \beta F \bar{\theta}^{2} / \gamma^{2}}$ and entry of the EG is deterred.

(iii) If $F \geq F^{\text {block, }}$, then the firm chooses $d^{*}(F)=d^{\text {block }}$ and entry of the $E G$ is blockaded.

\section{Proof See Appendix A.}

Proposition 2 characterizes for each value of $F$, the corresponding unique equilibrium value of the environmental damage per-unit of production chosen by the firm in stage 1 , and the subsequent entry decision of the EG in stage 2. Remark that for each case the subsequent unique equilibrium choice of the EG's effort in stage 2 and the firm's unique equilibrium price in stage 3 follow in a straightforward way. ${ }^{5}$

\section{Properties of the equilibrium}

In this section we discuss some interesting properties associated with the equilibrium of Proposition 2. We first focus on the environmental damage per-unit of production, the market demand for the product and the aggregated environmental damage. To begin with, we present an immediate corollary of Proposition 2.

Corollary 1 In the equilibrium the following holds for the environmental damage per-unit of production:

(i) $d^{*}(F)$ is a continuous function of $F$, except in the point $F^{\text {deter }}$ where it jumps downwards.

(ii) $d^{\text {acc }}<d^{\text {block }}$.

(iii) If $F^{\text {deter }} \leq F<F^{\text {block }}$, then $d^{*}(F)$ is strictly increasing in $F$ and $d^{*}(F)<d^{\text {block }}$.

Proof See Appendix A.

The interpretation of Corollary 1 is straightforward. Part (iii) shows that if $F \in$ $\left[F^{\text {deter }}, F^{\text {block }}\right)$, then the firm deters entry by lowering $d$ relative to $d^{\text {block }}$. Parts (i) and (iii) imply that there exists an $\tilde{F} \in\left(F^{\text {deter }}, F^{\text {block}}\right)$ such that $d^{*}(F)<d^{\text {acc }}$ if and only if $F \in\left[F^{\text {deter }}, \tilde{F}\right)$. Thus, if $F \in\left[F^{\text {deter }}, \tilde{F}\right)$, then the firm deters entry by lowering $d$ relative to $d^{a c c}$. In the latter case, there is less environmental damage per-unit of production than under entry by the EG. The Corollary is illustrated in Fig. 1.

Next, let $q^{*}(F)$ represent the equilibrium market demand as a function of $F$. Then for $0 \leq F<F^{\text {deter }}$, we have $q^{*}(F)=q^{a c c}$, where $q^{a c c}$ is obtained by substituting $d=d^{a c c}$ and $x=x^{e}\left(d^{a c c}\right)$ in (5). For $F^{\text {deter }} \leq F<F^{\text {block }}, q^{*}(F)$ follows from substitution of

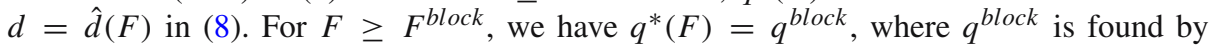
substituting $d=d^{\text {block }}$ in (8). We now can give the following result.

5 In Appendix B we present conditions (B.1), (B.2) and (B.4), which guarantee that Assumption 1 is satisfied in, respectively, the case of blockaded entry, entry deterrence, and entry accommodation. 


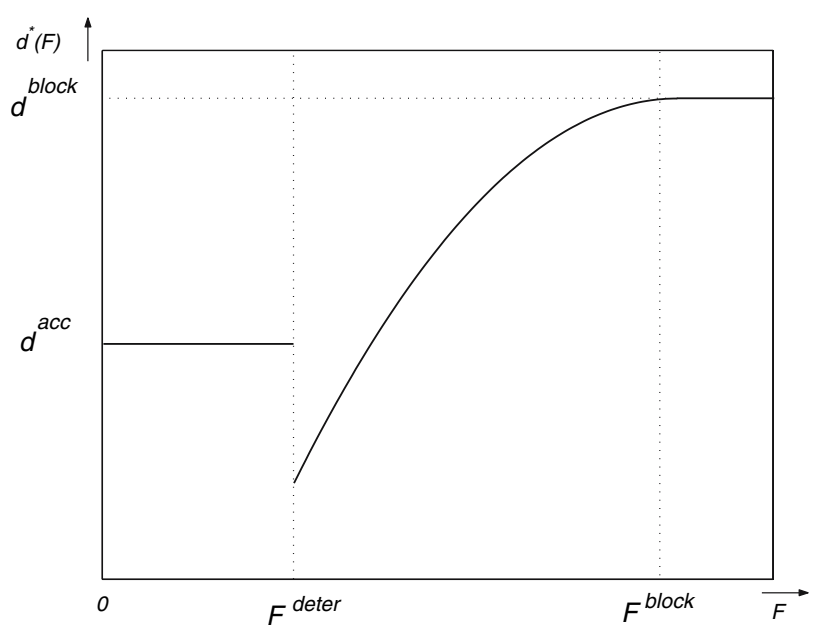

Fig. 1 Equilibrium environmental damage per-unit of production $d^{*}(F)$ as a function of $F$

Corollary 2 In the equilibrium the following holds for the demand of the firm:

(i) $q^{*}(F)$ is a continuous function of $F$, except in the point $F^{\text {deter }}$ where it jumps upwards.

(ii) $q^{\text {acc }}<q^{\text {block }}$.

(iii) If $F^{\text {deter }} \leq F<F^{\text {block}}$, then $q^{*}(F)$ is either strictly decreasing in $F$, or $q^{*}(F)$ is first strictly increasing in $F$ and next strictly decreasing in $F$.

Proof See Appendix A.

According to part (i) of this corollary, if the situation switches from entry accommodation to entry deterrence, then the demand for the product jumps upwards. This rise in demand originates from the jump downwards in the environmental damage per-unit of production, and the subsequent drop in the disutility of the environmental damage experienced by the consumers. Part (iii) shows that if $F$ increases from $F^{\text {deter }}$ to $F^{\text {block }}$, then from some point onwards the firm's demand will decrease to the level $q^{\text {block}}$. Notice that the decrease in the demand is caused by the fact that the increase in $F$ is accompanied by an increase in $d^{*}(F)=\hat{d}(F)$, which enlarges for each consumer the disutility of the environmental damage per-unit of production.

Proceeding, let $D^{*}(F)=q^{*}(F) d^{*}(F)$ denote the equilibrium aggregated environmental damage as a function of $F$. Obviously, for $0 \leq F<F^{\text {deter }}$ we have $D^{*}(F)=q^{\text {acc }} d^{a c c}$; for $F^{\text {deter }} \leq F<F^{\text {block }}$ we have $D^{*}(F)=\hat{q}(F) \hat{d}(F)$; and for $F \geq F^{\text {block }}$ we have $D^{*}(F)=q^{\overline{b l o c k}} d^{\text {block}}$. Using this notation, we can present the next proposition.

Proposition 3 In the equilibrium the following holds for the aggregated environmental damage:

(i) $D^{*}(F)$ is a continuous function of $F$ except in the point $F=F^{\text {deter }}$ where it jumps downwards.

(ii) $q^{\text {acc }} d^{\text {acc }}<q^{\text {block }} d^{\text {block }}$.

(iii) If $F^{\text {deter }} \leq F<F^{\text {block }}$, then $D^{*}(F)$ is strictly increasing in $F$.

(iv) If $F<F^{\text {deter }}$ or $F \geq F^{\text {block, }}$, then $D^{*}(F)$ is constant in $F$.

As a result, aggregated environmental damage reaches a minimum if $F=F^{\text {deter }}$. 
Proof See Appendix A.

In part (i) of Corollary 1 we have seen that the environmental damage per-unit of production jumps downwards in the point $F=F^{\text {deter }}$, whereas part (i) of Corollary 2 learned that the demand of the firm jumps upwards in this point. Interestingly, part (i) of Proposition 3 shows that the former effect dominates the latter; i.e., if the situation switches from entry accommodation to entry deterrence, then the aggregated environmental damage jumps downwards. Parts (i) and (iii) imply that the aggregated environmental damage reaches a minimum if $F=F^{\text {deter }}$. This, in turn, implies that in the case of entry deterrence there is an interval with $F$ small enough, on which aggregated environmental damage is lower than if the firm accommodates. Note that $D^{*}(F)$, qualitatively, is similar to $d^{*}(F)$. Hence, a plot of $D^{*}(F)$ would look very much like the function plotted in Fig. 1.

Concluding this section, we make three remarks with regard to the total costs of the EG, the firm's profit, and the consumer surplus and social welfare in the equilibrium for different values of $F$.

Remark 1 Let us represent the total equilibrium costs of the EG by

$$
\Delta^{*}(F) \equiv \begin{cases}\gamma q^{a c c} d^{a c c}+s\left(x^{e}\left(d^{a c c}\right)\right)+F & \text { if } 0 \leq F<F^{\text {deter }}, \\ \gamma q^{*}(F) d^{*}(F) & \text { if } F^{\text {deter }} \leq F<F^{\text {block }} \\ \gamma q^{\text {block }} d^{\text {block }} & \text { if } F \geq F^{\text {block }}\end{cases}
$$

It follows immediately that statements similar to parts (i) and (iii) of Proposition 3 also apply to the EG's total equilibrium costs: i.e., (a) $\Delta^{*}(F)$ is a continuous function of $F$ except in the point $F=F^{\text {deter }}$ where it jumps downwards; and (b) if $F^{\text {deter }} \leq F<F^{\text {block }}$, then $\Delta^{*}(F)$ is strictly increasing in $F$. Using part (i) of Proposition 3 it also follows that $\Delta^{*}(0)>\Delta^{*}\left(F^{\text {deter }}\right)$. Thus, the EG's total costs are larger in the case of entry accommodation (even with $F=0$ ) than in the entry deterrence case with $F=F^{\text {deter }}$. Finally, it can also be

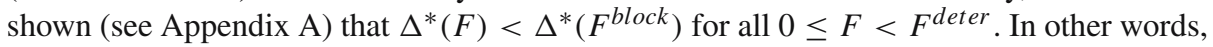
for the EG the total costs in the situation with blockaded entry are larger than in all possible situations with entry accommodation.

Remark 2 Considering the equilibrium profit of the firm, let $0 \leq F<F^{\text {deter }}$. For these values of $F$, the firm accommodates entry and sets $d^{*}(F)=d^{a c c}$. The EG reacts by setting $x=x^{e}\left(d^{a c c}\right)=\gamma d^{a c c} /(4 \beta \bar{\theta})$. The firm's profit is the same for all $F$ in the relevant region. Next, let $F^{\text {deter }} \leq F<F^{\text {block }}$. Then the firm deters entry by setting $d^{*}(F)=\hat{d}(F)$, which is an increasing function of $F$. Recall that $\hat{d}(F)<d^{\text {block }}$. Since $d^{\text {block }}$ is the unique and global maximum of $\Pi^{n e}$, the firm's profit must be an increasing function of $F$ in this region of $F$. Finally, take $F \geq F^{\text {block }}$. Then entry is blockaded, the firm sets $d=d^{\text {block }}$, and its profit is constant for all $F$ in this region. It is also easy to see that the equilibrium profit is a continuous function for all $F \geq 0$.

Remark 3 It seems tempting to examine the consumer surplus associated with the equilibrium as well. However, a consistent comparison of the size of the consumer surplus for different values of $F$ is hindered by the fact that the utility function of each consumer depends on $x$, which might take on different values depending on the size of $F$. In turn, this hinders an analysis of the social welfare properties of the equilibrium as well. The problematic nature of welfare analyses in models with endogenously shifting preferences is well-known in the literature on persuasive advertising, see Dixit and Norman (1978). Dixit and Norman's approach is the following: compare welfare before and after the change of preferences using 
both the unchanged and the changed preferences for the consumer surplus. If both measures point in the same direction, say upwards, then they argue that it is safe to say that advertising indeed increases social welfare. Their approach is, however, somewhat controversial, see Carlton and Perlof (2005, pp. 483-486). Simulations carried out by us suggests that, for most parameter values, it does not matter if pre- or post-campaign preferences are used to construct social welfare. In particular, it seems that social welfare is highest when entry is deterred. There are, however, exceptions. For instance, if $V=1, \alpha=1 / 4, \beta=1 / 4$, $\gamma=1 / 2$ and $\bar{\theta}=1$, then under pre-campaign preferences social welfare is highest if entry is accommodated, but under post-campaign preferences social welfare is highest if entry is deterred. ${ }^{6}$ This impedes application of the methodology of Dixit and Norman, and we conclude that further analysis of the social welfare properties of the equilibrium of our model is not meaningful.

\section{Conclusion}

This paper has analysed a market on which a monopolistic firm supplies an environmentally unfriendly good. The firm can influence the amount of damage generated per-unit of production of the good by choosing an appropriate production technique. However, a cleaner product also implies that the per-unit cost of production becomes higher. Consumers of the good dislike the environmental damage caused by the good, but they are heterogeneous with respect to the size of the associated disutility. The key feature of our analysis is that we assume that an EG can enter the market and set up a campaign in order to increase each consumer's disutility of the environmental damage caused by the consumption of the good. Thus, instead of analysing the effectiveness of traditional regulatory measures (like taxes on dirty goods or unit-emission standards, etcetera) imposed by a government, we focus here on the impact on the market outcome of the presence of a non-governmental organization like an EG.

We have derived the characteristics of the equilibrium of the resulting entry-deterrence game between the firm and the EG. It turned out that the aggregated environmental damage is lowest if the firm is able to deter entry of the EG and, moreover, the EG's fixed entry $\operatorname{cost} F$ is small enough. Hence, the threat of entry (instead of the actual entry) by the EG is most useful in this case. More technically, it appeared that aggregated environmental damage reaches a minimum if $F=F^{\text {deter }}$. Hence, our analysis suggests that it might be useful for a government, which is interested in minimal aggregated environmental damage, to commit itself prior to stage 1 of the game to the following policy measure towards an EG with entry cost $F$ : (a) if $F>F^{\text {deter }}$, then the EG receives a subsidy equal to $F-F^{\text {deter }}$ in case it actually sets up a campaign; (b) if $F<F^{\text {deter }}$, then the EG has to pay a tax equal to $F^{\text {deter }}-F$ in case it actually sets up a campaign. Observe that under this policy measure, in equilibrium the monopolist actually always deters entry of the EG. Hence, in equilibrium the government never has to pay a subsidy and never receives a tax, i.e., the measure is always budget neutral. This is, of course, a very simplistic view of the way governments subsidize EGs, but it does provide a rationale for these kind of subsidies.

We must emphasize that the current analysis still has a number of important caveats. We mention here three. First, we study the case in which there is a monopolistic firm. In a market with two firms the problem is changed in the following manner: if consumers want to buy the good, then the influence of the EG is also to direct these people to the cleanest product.

6 The example satisfies the conditions associated with Assumption 1 as discussed in Appendix B. 
That is, while the monopolistic firm only "competes" with the option of not consuming, an oligopolistic firm might also compete with the option that consumers buy another, cleaner, product from another firm. Therefore, there is an additional benefit from producing a clean good. If one firm decides to offer a cleaner product, then the other firms could well do the same thing. By focusing on a monopolistic market, and thus ignoring the strategic incentives firms have to supply a cleaner product than their rivals, it seems that, if anything, the effect of an EG is underestimated. Yet, it is interesting to study these issues in more detail in future research. Second, a specific formulation of the effect of the EG on the preferences of the consumers is employed. In particular, we assume that the disutility parameter of environmental damage per-unit of production increases with the same constant for all consumers. This implies that the mean disutility of the consumers increases but the variance remains constant. Interesting extensions would have the feature that the mean increases and, additionally, the variance will also change. This would reflect the idea that high or low disutility consumers are more or less susceptible to campaigning by EGs. We expect, however, that our main conclusion, i.e., the structure of the situation resembles an entry-deterrence game, will not be affected. Finally, advertising is modelled as being persuasive, i.e., the EG has the ability to shift preferences. While this type of advertising is relevant in practice, it impedes welfare comparisons.

Acknowledgements We thank two anonymous referees, Marco Haan, Peter Kooreman, John Maxwell and Eric Rasmusen for useful remarks and discussions. Pim Heynen gratefully acknowledges financial support from the Netherlands Organization for Scientific Research (NWO).

\section{Appendix A: proofs}

\section{Proof of Proposition 1}

Remark first that if $F=0$, then the EG will enter because we know that $x^{e}>0$. Next, notice that $x^{e}$ does not depend on $F$. Therefore, the LHS of (11) grows linearly in $F$ while the RHS is constant. This implies the existence of $\hat{F}(d)>0$. Clearly, $\hat{F}(d) \equiv \gamma\left(q^{n e}-q^{e}\right) d-s\left(x^{e}\right)=$ $\gamma x^{e} d /(2 \bar{\theta})-s\left(x^{e}\right)$. Since $x^{e}$ is the solution of $s^{\prime}(x)=\gamma d /(2 \bar{\theta})$, we have

$$
\frac{\mathrm{d} \hat{F}(d)}{\mathrm{d} d}=\frac{\gamma x^{e}}{2 \bar{\theta}}+\frac{\gamma d}{2 \bar{\theta}} \frac{\mathrm{d} x^{e}}{\mathrm{~d} d}-\frac{\mathrm{d} s}{\mathrm{~d} x} \frac{\mathrm{d} x^{e}}{\mathrm{~d} d}=\frac{\gamma x^{e}}{2 \bar{\theta}}>0,
$$

which completes the proof.

\section{Proof of Proposition 2}

We calculate the optimal $d$ for the cases of blockaded entry, entry deterrence and entry accommodation. In the case of blockaded entry, the firm maximizes $\Pi^{n e}$ with respect to $d$. The first-order condition is

$$
\frac{\mathrm{d} \Pi^{n e}}{\mathrm{~d} d}=\frac{2[V-c(d)]\left[-c^{\prime}(d) d\right]-[V-c(d)]^{2}}{4 \bar{\theta} d^{2}}=0 .
$$

Observe that this equation is satisfied if $d$ is chosen such that $V-c(d)=0$. However, such a choice of $d$ would result in zero profit and conflict with Assumption 1, and hence is not relevant. As a result, we can reduce (A.2) to

$$
V-c(d)+2 c^{\prime}(d) d=0,
$$

from which we obtain the corresponding unique optimal $d$, i.e.

$$
d^{\text {block }} \equiv \sqrt{\frac{5 \alpha}{V}}
$$


Using (A.3), it can be shown that the second-order derivative of $\Pi^{\text {ne }}$ with respect to $d$ evaluated in $d=d^{\text {block }}$ is negative if and only if $2 c^{\prime \prime}(d) d+c^{\prime}(d)>0$ for $d=d^{\text {block}}$. The latter inequality holds, since with $c(d)=\alpha / d^{2}$ we have for any $d>0$ that

$$
2 c^{\prime \prime}(d) d+c^{\prime}(d)=\frac{12 \alpha}{d^{3}}-\frac{2 \alpha}{d^{3}}=\frac{10 \alpha}{d^{3}}>0 .
$$

Hence, the second-order condition for a maximum is satisfied in $d=d^{\text {block }}$.

Substituting $d^{\text {block }}$ into (12), we obtain the corresponding threshold of the entry cost

$$
F^{\text {block }} \equiv \frac{5 \alpha \gamma^{2}}{16 \beta V \bar{\theta}^{2}}
$$

Concluding, if $F \geq F^{\text {block}}$, then there is blockaded entry. That is, the EG's entry cost is so high that the firm can simply choose the profit-maximizing value $d^{\text {block }}$ it would also choose if the EG would not consider entry at all; in turn, given $d^{\text {block}}$, it is indeed optimal for the EG to choose no entry.

Considering the case of entry deterrence, then the firm deliberately lowers the environmental damage to a point where the EG does not enter. Recalling (12), remark that if both $F<\hat{F}\left(d^{\text {block}}\right)$ and the firm ignored the possibility of entry and would set $d=d^{\text {block }}$, then the EG would enter, i.e., entry is not blockaded. The firm could, however, try to lower the environmental damage $d$ such that $F \geq \hat{F}(d)$, because in that case the EG has no reason to enter. Since $d^{\text {block }}$ is the global (and unique) maximum of the profit function $\Pi^{\text {ne }}(d)$, this means that deviating from $d^{\text {block }}$ lowers $\Pi^{n e}(d)$. In fact, the further $d$ is from $d^{\text {block }}$, the lower is the firm's profit. This implies that the firm will lower $d$ to the point where $\hat{F}(d)=F$, making the EG just indifferent between entering and not entering. We denote this level of environmental damage as $\hat{d}(F)$, and observe that it is given by

$$
\hat{d}(F) \equiv \sqrt{\frac{16 \beta F \bar{\theta}^{2}}{\gamma^{2}}} .
$$

Notice also that $\hat{F}(\hat{d}(F))=F$, and that for $F<F^{\text {block }}$ we have $\hat{d}(F)<d^{\text {block }}$. Of course, this behavior should be profitable for the firm, i.e., accommodating entry should give a lower profit. Whether this indeed is the case, depends on the magnitude of $F$. Before deriving the exact range of values of $F$ for which the firm will deter entry, it is convenient to consider first the case of entry accommodation.

In the case of entry accommodation, the firm maximizes $\Pi^{e}$ with respect to $d$. The firstorder condition reads

$$
\frac{\mathrm{d} \Pi^{e}}{\mathrm{~d} d}=\frac{2 d\left[V-c(d)-x^{e} d\right]\left[-c^{\prime}(d)-(\gamma d) /(2 \beta \bar{\theta})\right]-\left[V-c(d)-x^{e} d\right]^{2}}{4 \bar{\theta} d^{2}}=0 .
$$

This equation is satisfied if $d$ is chosen such that $V-c(d)-x^{e} d=0$. However, this choice of $d$ would result in zero profit and conflict with Assumption 1, and hence is not relevant. Thus, we can reduce (A.8) to

$$
\frac{5 \alpha}{d^{2}}=V+\frac{3 \gamma d^{2}}{4 \beta \bar{\theta}}
$$

which can be rewritten as a quadratic equation in $d^{2}$. From this, one can verify that (A.9) has a unique positive solution, which we denote as $d^{a c c}>0$. After a straightforward calculation 
it can be shown that the second-order derivative of $\Pi^{e}$ with respect to $d$ evaluated in $d=d^{a c c}$ is negative if and only if

$$
2 c^{\prime \prime}(d) d+c^{\prime}(d)+\frac{3 \gamma d}{2 \beta \bar{\theta}}>0
$$

for $d=d^{a c c}$. Recalling (A.5), it follows directly that this is true, and we thus know that the second-order condition for a maximum is satisfied in $d=d^{a c c}$. Remarking that $d^{\text {block }}$ solves $5 \alpha / d^{2}=V$, it follows from (A.9) that $5 \alpha /\left(d^{\text {block }}\right)^{2}<5 \alpha /\left(d^{a c c}\right)^{2}$, and thus $d^{a c c}<d^{\text {block}}$. Concluding, the firm chooses $d^{a c c}$ if it accommodates to entry of the EG. Whether this behaviour is indeed optimal for the firm, depends on the size of $F$.

Therefore, let us now analyse for which values of $F$ either entry deterrence or entry accommodation is optimal for the firm. We make a number of observations. First, using (6) and (9), it follows that $\Pi^{e}(d)<\Pi^{n e}(d)$, i.e., given $d$, the firm's profit always decreases if the EG enters (of course, assuming that Assumption 1 holds). Second, we define $d^{0}>0$ according to $\Pi^{\text {ne }}\left(d^{0}\right)=0$. Using (9), it follows that $d^{0}=\sqrt{\alpha / V}$. Given $d^{0}$ and using (12), we next define $F^{0} \equiv \hat{F}\left(d^{0}\right)$, and notice that $F^{0}=\alpha \gamma^{2} /\left(16 \beta V \bar{\theta}^{2}\right)$. Remark that if the firm chooses $d=d^{0}$, then it is able to deter entry of the EG for $F \geq F^{0}$, and in that case its profit is just equal to zero. Third, observe that $\Pi^{n e}\left(d^{0}\right)=0<\Pi^{e}\left(d^{a c c}\right)<\Pi^{\text {ne }}\left(d^{\text {block }}\right)$. The last inequality follows since, while taking $d=d^{\text {block }}$, our first observation implies that $\Pi^{n e}(d)$ evaluated in its global maximum must be larger than $\Pi^{e}(d)$ evaluated in any $d$. Fourth, $\Pi^{\text {ne }}(d)$ is a continuous and strictly increasing function on the interval $\left[d^{0}, d^{\text {block }}\right]$. Fifth, combining results, we see that there exists a unique, $d^{\text {deter }}$ say, such that both $d^{0}<$ $d^{\text {deter }}<d^{a c c}$ and $\Pi^{n e}\left(d^{\text {deter }}\right)=\Pi^{e}\left(d^{a c c}\right)$. Remark that the firm is indifferent between choosing $d=d^{\text {deter }}$ (in which case there is entry deterrence) and $d=d^{a c c}$ (in which case there is entry accommodation). Given $d^{\text {deter }}$ and using (12), we define $F^{\text {deter }} \equiv \hat{F}$ ( $d^{\text {deter }}$ ), and notice that $F^{0}<F^{\text {deter }}<F^{\text {block }}$. Note also that $d^{*}\left(F^{\text {deter }}\right)=d^{\text {deter }}$. It follows that for the firm, it is profitable to deter entry if $F^{\text {deter }} \leq F<F^{\text {block}}$, whereas it is profitable to accommodate entry if $0 \leq F<F^{\text {deter }}$.

\section{Proof of Corollary 1}

Part (ii) is already discussed in the proof of Proposition 2. From that proof we also know that $d^{*}\left(F^{\text {deter }}\right)=d^{\text {deter }}<d^{a c c}$. Part (i) follows directly. Part (iii) follows from (A.7) and (ii) of Proposition 2.

\section{Proof of Corollary 2}

In order to prove part (i) we recall from the proof of Proposition 2 that $\Pi^{\text {ne }}\left(d^{\text {deter }}\right)=$ $\Pi^{e}\left(d^{a c c}\right)$. Using (5), (6), (8) and (9), we then obtain that $q^{\text {deter }}=q^{a c c} \sqrt{d^{a c c} / d^{\text {deter }}}$. Using $d^{\text {deter }}<d^{a c c}$, we thus see that $q^{\text {acc }}<q^{\text {deter }}$. Part (i) follows easily.

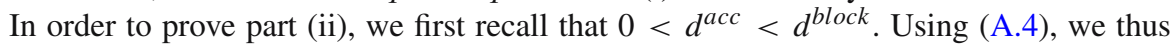
observe that we can write $d^{a c c}=\sqrt{k \alpha / V}$, with $k$ some real number satisfying $0<k<5$. Substituting this expression for $d^{a c c}$ in (A.9), and rearranging terms, we obtain

$$
\frac{\gamma}{\beta \bar{\theta}}=\frac{4((5 / k)-1) V^{2}}{3 k \alpha} \text {. }
$$

From this, we derive that

$$
x^{e}\left(d^{a c c}\right)=\frac{\gamma d^{a c c}}{4 \beta \bar{\theta}}=\frac{((5 / k)-1) V^{2}}{3 k \alpha} \times \sqrt{\frac{k \alpha}{V}} .
$$


Next, using (5) and (8), we observe that $q^{a c c}<q^{\text {block }}$ is equivalent with

$$
\frac{V-c\left(d^{a c c}\right)}{d^{a c c}}-\frac{V-c\left(d^{\text {block }}\right)}{d^{\text {block }}}<x^{e}\left(d^{\text {dacc }}\right) .
$$

Substituting $d^{a c c}=\sqrt{k \alpha / V}$ and (A.12) in (A.13), and multiplying the resulting expression on both sides with $\sqrt{(\alpha / V)} / V$, we obtain

$$
\frac{1-(1 / k)}{\sqrt{k}}-\frac{4}{5 \sqrt{5}}<\frac{((5 / k)-1) \sqrt{k}}{3 k}
$$

which after some straightforward manipulations can be rewritten as

$$
(k-2) \frac{1}{k \sqrt{k}}<\frac{3}{5 \sqrt{5}},
$$

which is satisfied for all $0<k<5$. This establishes part (ii).

In order to prove part (iii), we observe that for $F \in\left[F^{\text {deter }}, F^{\text {block }}\right)$ we have

$$
\frac{\mathrm{d} q^{*}(F)}{\mathrm{d} F}=\frac{\mathrm{d} q^{n e}(d)}{\mathrm{d} d} \cdot \frac{\mathrm{d} \hat{d}(F)}{\mathrm{d} F} .
$$

The second term on the RHS is positive, while the first term is positive if and only if $\hat{d}(F)<\sqrt{3 \alpha / V}$, i.e. using (A.7), if and only if $F<3 \alpha \gamma^{2} /\left(16 \beta \bar{\theta}^{2} V\right)$. We recall from the proof of Proposition 2 that $F^{\text {deter }} \in\left(F^{0}, F^{\text {block}}\right)$, where $F^{0}=\alpha \gamma^{2} /\left(16 \beta \bar{\theta}^{2} V\right)$ and $F^{\text {block }}=5 \alpha \gamma^{2} /\left(16 \beta \bar{\theta}^{2} V\right)$. We observe that $F^{\text {deter }}$ can be either smaller or larger than $3 \alpha \gamma^{2} /\left(16 \beta \bar{\theta}^{2} V\right)$. For example, if $\alpha=0.05, \beta=\gamma=\bar{\theta}=V=1$, then $F^{\text {deter }}=0.0087<0.0094=3 \alpha \gamma^{2} /\left(16 \beta \bar{\theta}^{2} V\right)$. But, if we take $\bar{\theta}=2$ keeping the other parameters the same, we have $F^{\text {deter }}=0.0026>0.0023=3 \alpha \gamma^{2} /\left(16 \beta \bar{\theta}^{2} V\right)$. (Both examples satisfy the conditions associated with Assumption 1 as discussed in Appendix B.) Part (iii) follows directly.

\section{Proof of Proposition 3}

First recall from the proof of Proposition 2 that $\Pi^{\text {ne }}\left(d^{\text {deter }}\right)=\Pi^{e}\left(d^{a c c}\right)$. From this and (6) and (9), we obtain

$$
\left[V-c\left(d^{a c c}\right)-x^{e}\left(d^{a c c}\right) d^{a c c}\right]=\left[V-c\left(d^{\text {deter }}\right)\right] \sqrt{\frac{d^{a c c}}{d^{\text {deter }}}} .
$$

Recalling that $d^{\text {deter }}<d^{a c c}$, we thus have

$$
\left[V-c\left(d^{a c c}\right)-x^{e}\left(d^{a c c}\right) d^{a c c}\right]>\left[V-c\left(d^{\text {deter }}\right)\right] .
$$

Using (5) and (8), we can conclude that $q^{a c c} d^{a c c}>q^{\text {deter }} d^{\text {deter }}$. Part (i) follows easily. have

In order to prove part (ii), observe that since $\Pi^{e}\left(d^{a c c}\right)=\Pi^{\text {ne }}\left(d^{\text {deter }}\right)<\Pi^{\text {ne }}\left(d^{\text {block }}\right)$, we

$$
\left[V-c\left(d^{a c c}\right)-x^{e}\left(d^{a c c}\right) d^{a c c}\right]<\left[V-c\left(d^{\text {block }}\right)\right] \sqrt{\frac{d^{a c c}}{d^{b l o c k}}} .
$$

Using $d^{a c c}<d^{\text {block}}$, (5) and (8), we obtain that $q^{a c c} d^{a c c}<q^{\text {block }} d^{\text {block }}$, i.e. part (ii).

In order to prove part (iii), let $F^{\text {deter }} \leq F<F^{\text {block}}$. Using (8), we see that for such $F$ we have $D^{*}(F)=q^{*}(F) d^{*}(F)=(V-c(\hat{d}(F))) /(2 \bar{\theta})$. We next observe that this $D^{*}(F)$ is increasing in $F$ because

$$
\frac{\mathrm{d} D^{*}(F)}{\mathrm{d} F}=\frac{1}{2 \bar{\theta}}\left[\frac{\mathrm{d}}{\mathrm{d} F}(V-c(\hat{d}(F)))\right]=\frac{1}{2 \bar{\theta}}\left[-c^{\prime}(\hat{d}(F))\right]>0 .
$$


This completes the proof.

\section{Ad Remark 1}

Take an arbitrary $0 \leq F<F^{\text {deter }}$. We know that given such an $F$, in equilibrium we have $d=d^{a c c}$, and moreover

$$
\begin{aligned}
\Delta^{*}(F) & =\gamma q^{a c c} d^{a c c}+s\left(x^{e}\left(d^{a c c}\right)\right)+F \\
& <\gamma q^{n e}\left(d^{a c c}\right) d^{a c c} \\
& <\gamma\left(\frac{V-c\left(d^{a c c}\right)}{2 \bar{\theta}}\right) \\
& <\gamma\left(\frac{V-c\left(d^{\text {block }}\right)}{2 \bar{\theta}}\right) \\
& =\Delta^{*}\left(F^{\text {block }}\right),
\end{aligned}
$$

where the first inequality follows since entry is profitable for the EG if $d=d^{a c c}$, the second inequality uses (8), and the third one uses $d^{a c c}<d^{\text {block }}$.

\section{Appendix B: details regarding assumption 1}

In deriving the equilibrium of the game, we have acted so far as if Assumption 1 simultaneously holds for $d^{\text {block }}$ (blockaded entry), $\hat{d}(F)$ (entry deterrence) and $d^{a c c}$ and $x^{e}=x^{e}\left(d^{a c c}\right)$ (entry accommodation). In this appendix we will show that there indeed exist combinations of parameters of the model for which our approach is justified. We will examine the three cases one by one. Again we use $c(d)=\alpha / d^{2}$ and $s(x)=\beta x^{2}$, with $\alpha>0$ and $\beta>0$.

Blockaded entry It has to be shown that there exist parameters such that $V-c(d)>0$ and $V-c(d)<2 \bar{\theta} d$ for $d=d^{\text {block}}$. Substituting (A.4), it follows that the first inequality is always satisfied whereas the second inequality is satisfied if and only if

$$
\alpha>\frac{4 V^{3}}{125 \bar{\theta}^{2}} \text {. }
$$

Entry deterrence We have to demonstrate that there exist parameters such that $V-c(d)>0$ and $V-c(d)<2 \bar{\theta} d$ for $d=\hat{d}(F)$. Substituting (A.7), we see that the first inequality is satisfied if and only if

$$
\alpha<\frac{16 \beta F V \bar{\theta}^{2}}{\gamma^{2}} .
$$

Next, taking arbitrary $d>0$ and substituting $c(d)=\alpha / d^{2}$, we can rewrite $V-c(d)<2 \bar{\theta} d$ as

$$
2 \bar{\theta} d^{3}-V d^{2}+\alpha>0 .
$$

Clearly, (B.3) is satisfied if $d$ is close enough to zero and if $d$ becomes infinitely large. Furthermore, the LHS of (B.3) has a minimum at $d=V /(3 \bar{\theta})$. Substitution shows that condition (B.3) evaluated in this minimum is satisfied if and only if

$$
\alpha>\frac{V^{3}}{27 \bar{\theta}^{2}} .
$$


Clearly, (B.4) is sufficient to guarantee that $V-c(d)<2 \bar{\theta} d$ for $d=\hat{d}(F)$.

Accommodation We have to show that $V-c(d)-x d>0$ and $V-c(d)-x d<2 \bar{\theta} d$ for $d=d^{a c c}$ and $x=x^{e}=x^{e}\left(d^{a c c}\right)$. Observe that $d^{a c c}$ is defined as the solution of $-2 c^{\prime}(d) d=V-c(d)+3 x^{e} d$, which can be rewritten as

$$
\begin{aligned}
V-c(d)-x^{e} d & =-2 c^{\prime}(d) d-4 x^{e} d \\
& =\frac{4 \alpha}{d^{2}}-\frac{\gamma d^{2}}{\beta \bar{\theta}} .
\end{aligned}
$$

Therefore, instead of showing that $V-c(d)-x^{e} d>0$ for $d=d^{a c c}$, we can also show that

$$
\left(d^{a c c}\right)^{2}<\sqrt{\frac{4 \alpha \beta \bar{\theta}}{\gamma}} .
$$

Next, recall from (A.9) that $d^{a c c}$ is the solution of

$$
\frac{3 \gamma}{4 \beta \bar{\theta}} d^{4}+V d^{2}-5 \alpha=0,
$$

which is a quadratic equation in $d^{2}$. Solving this equation for $d^{2}$, and picking out the positive solution, we obtain that

$$
\left(d^{a c c}\right)^{2}=\sqrt{\left(\frac{2 \beta \bar{\theta} V}{3 \gamma}\right)^{2}+\frac{60 \alpha \beta \bar{\theta}}{9 \gamma}}-\frac{2 \beta \bar{\theta} V}{3 \gamma} .
$$

The aim is now to show under which conditions we have

$$
\sqrt{\left(\frac{2 \beta \bar{\theta} V}{3 \gamma}\right)^{2}+\frac{60 \alpha \beta \bar{\theta}}{9 \gamma}}-\frac{2 \beta \bar{\theta} V}{3 \gamma}<\sqrt{\frac{4 \alpha \beta \bar{\theta}}{\gamma}} .
$$

Therefore, define $A \equiv 2 \beta \bar{\theta} V /(3 \gamma), B \equiv 20 \beta \bar{\theta} /(3 \gamma)$ and $C \equiv 4 \beta \bar{\theta} / \gamma$, where $B>C$. Using these definitions and applying straightforward manipulations, we can rewrite (B.10) as

$$
\alpha<\frac{4 A^{2} C}{(B-C)^{2}}
$$

which is equivalent with

$$
\alpha<\frac{\beta \bar{\theta} V^{2}}{\gamma} .
$$

Finally, we need to show that for $d=d^{a c c}$ we have $V-c(d)-x^{e} d<2 \bar{\theta} d$, which, using (B.5), is equivalent with $-2 c^{\prime}(d) d<2 \bar{\theta} d+4 x^{e} d$. Using $d^{\text {block }}>d^{a c c}$, it follows that $c^{\prime}\left(d^{a c c}\right)>c^{\prime}\left(d^{\text {block }}\right)>-\bar{\theta}$. The latter inequality follows since $d^{\text {block }}$ is defined by (A.3) and, moreover, we know that $V-c\left(d^{\text {block }}\right)<2 \bar{\theta} d^{\text {block }}$. Using $c^{\prime}\left(d^{a c c}\right)>-\bar{\theta}$, we have $-2 c^{\prime}\left(d^{a c c}\right) d^{a c c}<2 \bar{\theta} d^{a c c}$, and thus certainly $-2 c^{\prime}\left(d^{a c c}\right) d^{a c c}<2 \bar{\theta} d^{a c c}+4 x^{e} d^{a c c}$.

Conclusion There are four restrictions, namely (B.1), (B.2), (B.4) and (B.12). Two of these are lower bounds on $\alpha$ and the other two are upper bounds on $\alpha$. Remark that all restrictions are satisfied if, for example, we take $\beta$ or $\bar{\theta}$ large enough, or $\gamma$ small enough. Hence, the restrictions are not contradictory. 


\section{References}

Arora S, Gangopadhyay S (1995) Toward a theoretical model of emissions control. J Econ Behav Organ 28:289-309

Bagwell K (2005) The economic analysis of advertising. In: Armstrong M, Porter RH (eds) Forthcoming in: The handbook of industrial organization, vol 3. North-Holland, Amsterdam

Bansal S, Gangopadhyay S (2003) Tax/subsidy policies in the presence of environmentally aware consumers. J Environ Econ Manage 45:333-355

Bjørner TB, Hansen LG, Russell CS (2004) Environmental labeling and consumers' choice - an empirical analysis of the effect of the Nordic swan. J Environ Econ Manage 47:411-434

Bloch F, Manceau D (1999) Persuasive advertising in Hotelling's model of product differentiation. Int J Indus Organ 17:557-574

Brockmann KL, Hemmelskamp J, Hohmeyer O (1996) Certified tropical timber and consumer behaviour. Centre for European-Economic Research, Physica Verlag, Heidelberg, Germany

Carlton DW, Perloff JM (2005) Modern industrial organization. 4th edn. Pearson, Addison Wesley, Boston

Cremer H, Thisse J-F (1999) On the taxation of polluting products in a differentiated industry. Eur Econ Rev 43:575-594

Dixit A, Norman V (1978) Advertising and welfare. Bell J Econ 9:1-17

von der Fehr N-H, Stevik K (1998) Persuasive advertising and product differentiation. South Econ J 65:113126

Grossman GM, Helpman E (2001) Special interest politics. MIT Press, Cambridge, MA

Heijnen P (2007) Informative advertising by an environmental group, CeNDEF working paper 07-02, University of Amsterdam (see www1.fee.uva.nl/cendef/publications/)

Hertzendorf MN, Overgaard PB (2001) Price competition and advertising signals: signaling by competing senders. J Econ Manage Strategy 10:621-662

Heyes AG (1997) Environmental regulation by private contest. J Public Econ 63:407-428

Levitt SD, Dubner SJ (2005) Freakonomics. Penguin Group Ltd, London

Liston-Heyes C (2001) Setting the stakes in environmental contests. J Environ Econ Manage 41:1-12

Maxwell JW, Lyon TP, Hackett SC (2000) Self-regulation and social welfare: the political economy of corporate environmentalism. J Law Econ 43:583-617

Milgrom P, Roberts J (1986) Price and advertising, signals of product quality. J Political Econ 94:796-821

Moraga-González JL, Padrón-Fumero N (2002) Environmental policy in a green market. Environ Resour Econ 22:419-447

Teisl MF, Roe B, Hicks RL (2002) Can eco-labels tune a market? Evidence from dolphin-safe labeling. J Environ Econ Manage 43:339-360

Tirole J (1988) The theory of industrial organization. MIT Press, Cambridge, MA

Tremblay VJ, Martins-Filho C (2001) A model of vertical differentiation, brand loyality, and persuasive advertising. In: Baye MR, Nelson JP (eds) Advances in applied microeconomics, vol 10. JAI Elsevier, Amsterdam 